\title{
Alterações na vegetação herbácea de floresta ombrófila densa decorrentes do uso em uma trilha turística na Serra do Mar em São Paulo, Brasil
}

\author{
Luísa Almeida Maciel ${ }^{1,2}$, Maria Francisca Roncero Siles ${ }^{1}$ e Marisa Dantas Bitencourt ${ }^{1}$
}

Recebido em 28/01/2010. Aceito em 17/06/2011

\begin{abstract}
RESUMO
(Alterações na vegetação herbácea de floresta ombrófila densa decorrentes do uso em uma trilha turística na Serra do Mar em São Paulo, Brasil). O estudo analisou a vegetação herbácea ao longo de uma trilha de uso público intensivo nos parques Natural Municipal Nascentes de Paranapiacaba (PNMNP) e Estadual da Serra do Mar (PESM). Foram analisados os valores de cobertura vegetal, riqueza e número de indivíduos do estrato herbáceo. A metodologia permitiu amostrar 76 unidades (adjacentes a trilha, a $2 \mathrm{~m}$ e a $10 \mathrm{~m}$ da trilha). Os três parâmetros da vegetação analisados apresentaram valores menores à medida que se afasta perpendicularmente à trilha. Na análise dos indivíduos registrou-se maior número de morfoespécies, maiores cobertura e riqueza nas unidades amostrais adjacentes à trilha. Duas espécies foram detectadas como dominantes, especialmente nas unidades amostrais próximas à trilha: Parodiolyra micrantha e Impatiens wallerianna. Esta última espécie encontra-se na lista oficial de espécies invasoras do município de São Paulo.
\end{abstract}

Palavras-chave: Mata Atlântica, recreação, uso público, trilhas, Unidades de Conservação

\begin{abstract}
(Alterations in the herbaceous vegetation along a tourist trail in the Serra do Mar, São Paulo, Brazil). This study analyzed the herbaceous vegetation along a publically used trail that crosses two important parks: Natural Municipal Nascentes de Paranapiacaba (PNMNP) and Estadual da Serra do Mar (PESM). The cover percentage, richness and number of individuals of the herbaceous layer were analyzed based on 76 samples (collected next to, $2 \mathrm{~m}$ and $10 \mathrm{~m}$ from the trail, perpendicularly). The values of the three dependent variables analyzed were smaller in the vegetation that was further ( 2 and $10 \mathrm{~m}$ ) from the trail. The analyses of the individuals sampled showed a higher number of morpho-species, and larger cover and richness, closer the trail. Parodiolyra micrantha and Impatiens wallerianna (which is officially listed as invasive in the Sao Paulo municipality) were dominant, especially closer to the trail.
\end{abstract}

Key words: Atlantic forest, Recreation Ecology, public use, trail, Natural Protected Areas

\section{Introdução}

Trilhas e caminhos, historicamente essenciais ao homem na constante busca de alimento e água, são hoje de grande importância para a recreação em áreas naturais ao redor do mundo, seja por fornecer acesso a pontos atrativos como mirantes e cachoeiras, ou simplesmente por oferecer ao visitante bem estar e lazer durante uma caminhada e a oportunidade de alcançar maior familiaridade com o meio natural.

O Brasil, país detentor da maior biodiversidade do planeta (Brasil 1998), vem experimentando um aumento da visitação em áreas naturais. Desse modo, emerge uma necessidade de pesquisas ecológicas visando subsídios que contribuam para minimizar o conflito entre uso e preservação (Kinker 1999; Cole 1978).

Em trilhas, pode ocorrer destruição da cobertura vegetal, diminuição da diversidade de espécies, deslocamento, e rearranjo na estrutura das comunidades vegetais (Cole 1978; Hammitt \& Cole 1998; Roncero-Siles 2003). A vegetação nativa e a serapilheira, que provém umidade e nutrientes ao solo, podem ser removidas com o pisoteio, o que altera a dinâmica original. Cole (1978) investigou alterações na cobertura vegetal e composição florística em oito vegetações

\footnotetext{
1 Universidade de São Paulo, Departamento de Ecologia do Instituto de Biociências São Paulo, SP, Brasil

2 Autora para correspondência: luisa.maciel@usp.br
} 
diferentes de sub-bosque, e observou redução nos valores de cobertura, assim como variações significativas na composição florística nas comunidades adjacentes às trilhas. Estudo realizado em florestas tropicais da Costa Rica indicou aumento em índices de diversidade e cobertura vegetais em amostras mais afastadas da trilha (Boucher et al.1991).

Este estudo vem de encontro com as atuais necessidades em se conciliar o uso público com os demais objetivos das Unidades de Conservação, que de acordo com Sun \& Walsh (1998), trazendo um enriquecimento da base teórica sobre os principais impactos ambientais decorrentes desse uso, de modo a minimizá-los e auxiliar o manejo dos recursos.

Já existe literatura substancial acerca dos impactos humanos sobre solo e vegetação, entretanto, poucos trabalhos foram desenvolvidos em florestas pluviais tropicais (Boucher et al. 1991).

O objetivo geral do presente estudo foi analisar o efeito de uma trilha de uso intensivo sobre a vegetação herbácea em duas Unidades de Conservação de proteção integral como uma maneira de compreender os impactos sofridos por esse estrato da vegetação. Foram testadas duas hipóteses: a) a cobertura vegetal, a riqueza de espécies e o número de indivíduos do estrato herbáceo aumentam conforme ocorre o afastamento da trilha; e b) há diferenças nas variáveis medidas entre os dois lados da trilha.

\section{Material e métodos}

O estudo foi desenvolvido na Trilha do Mirante, em fragmento florestal de Mata Atlântica na Serra do Mar, com predomínio de Floresta Ombrófila Densa. O percurso se inicia no Parque Natural Municipal Nascentes de Paranapiacaba - PNMNP (município de Santo André) e termina no mirante, que está localizado no Parque Estadual da Serra do Mar - PESM (núcleo Cubatão). Ambas são Unidades de Conservação de Proteção Integral, situadas na Serra do Mar. Nas proximidades, encontra-se a Reserva Biológica do Alto da Serra de Paranapiacaba - RBASP, também de proteção integral. As três unidades estão inseridas na Reserva da Biosfera do Cinturão Verde da Cidade de São Paulo, assim como à Reserva da Biosfera da Mata Atlântica, ambas protegidas pela UNESCO. A área de estudo, portanto, está inserida em um rico contexto de Unidades de Conservação no Bioma Mata Atlântica.

De acordo com o zoneamento preliminar do Parque das Nascentes, a Trilha do Mirante encontra-se na zona de recuperação (Santo André 2008). Com extensão de 1.185 m e situado a aproximadamente $975 \mathrm{~m}$ de altitude, o percurso é considerado de baixa dificuldade (declividade média de $15^{\circ}$ ) e leva em torno de uma hora para ser feito. A Trilha do Mirante está classificada como em situação prioritária de adequação ao uso ao uso público (LUME 1999). Escorregamentos ou deslizamentos de terra são freqüentes, particularmente no período chuvoso (UMAH 2000 apud Santo André 2008). Esta trilha era antigamente utilizada para a manutenção das primeiras antenas de TV da Serra do Mourão, apresentando muitos trechos em que sua largura supera o metro (Santo André 2008).

Para o estudo da vegetação foi utilizada a mesma metodologia empregada por Dale \& Weaver (1974); Cole (1978); Aspinall \& Pye (1987); Boucher et al. (1991); Roovers et al. (2004). Foi projetada uma amostragem em 18 pontos ao longo da trilha, separados por $40 \mathrm{~m}$. Em cada ponto foi prevista uma transeção com 6 unidades amostrais de $0,25 \mathrm{~m}^{2}$ sendo dois na vegetação adjacente à trilha, dois à distância de $2 \mathrm{~m}$ e os últimos a $10 \mathrm{~m}$ da borda trilha (figura 1). Os quadrados a $10 \mathrm{~m}$ foram considerados controle, por estarem a uma distância não influenciada pelo impacto ocorrido na trilha (Cole 1978; Dale \& Weaver 1974).

Foram estudados espécimes de altura inferior a $50 \mathrm{~cm}$ pertencentes ao estrato herbáceo, incluindo plantas herbáceas e plântulas de espécies lenhosas. As variáveis mensuradas foram: cobertura vegetal (estimada visualmente, sendo expressa em porcentagem), riqueza de morfoespécíes e número de indivíduos em cada quadrado (as espécies Philodendron e Parodiolyra foram consideradas indivíduos independentes sempre que havia enraizamento). Exemplares coletados foram levados ao Instituto de Botânica de São Paulo para herborização e identificação de espécies. Como a maioria dos espécimes coletados não estava em estado fértil, recorreu-se à classificação em famílias e morfoespécies.

As variáveis estudadas foram analisadas frente aos fatores independentes: distância $(0,2$ ou $10 \mathrm{~m})$ e lado (esquerdo ou direito) da trilha. Vale ressaltar que o lado direito da trilha apresentava um aclive, enquanto o lado esquerdo um

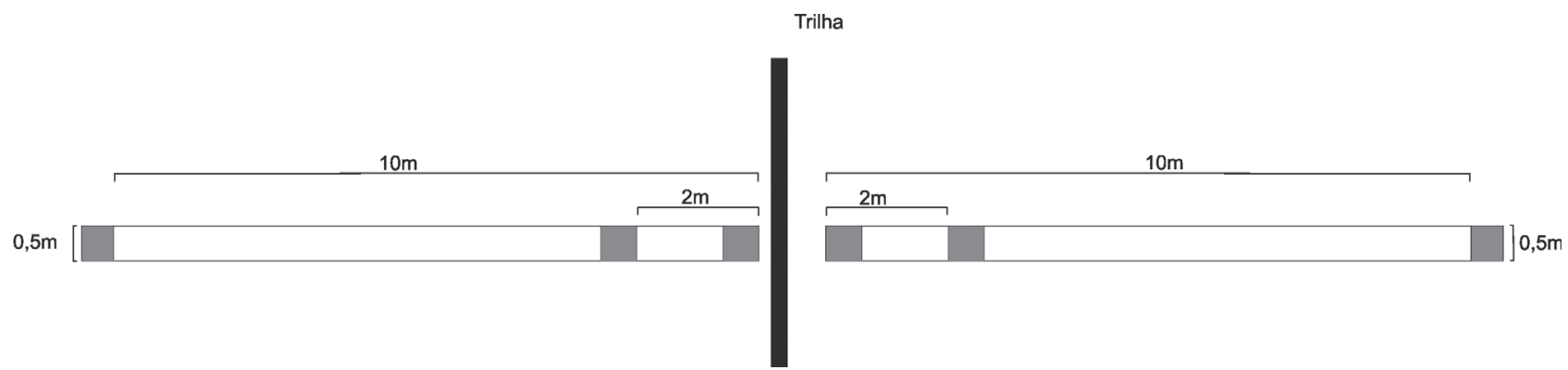

Figura 1. Disposição dos transectos perpendiculares à trilha e as medidas de distância e tamanho as unidades amostrais. 
declive. Foi estudado o efeito da distância, do lado da trilha e ainda da interação dos mesmos sobre as variáveis por meio da Análise de Variância (ANOVA de dois fatores) seguida de teste a posteriori Tuckey HSD (honestly significant difference) quando pertinente (Sokal \& Rohlf 1969).

\section{Resultados}

Em virtude de as condições físicas encontradas ao longo da trilha impedirem o acesso (como terreno muito íngreme, bifurcações, bambuzais densos e/ou um afloramento rochoso), não foi possível amostrar todos os quadrados inicialmente previstos. Ao todo, foram amostrados 76 quadrados de $0,25 \mathrm{~m}^{2}$ distribuídos entre 18 dezoito pontos (em cada ponto, perpendicularmente a trilha, nos dois lados, deveria se ter amostrado 6 quadrados: 18 x $6=108$ ).

Foram encontradas diferenças significativas nas três variáveis estudadas de acordo com a distância em relação à borda da trilha (figura 2), especificamente entre amostras adjacentes e as demais. Cobertura vegetal: ANOVA: $\mathrm{F}=6$, 8305; $p<<0,05$; Tuckey: $p<0,05$; riqueza: ANOVA: $\mathrm{F}=13$, 7584; $p<<0,05$; Tuckey: $p<0,05$; e número de indivíduos: ANOVA: $\mathrm{F}=19,5316 ; p<<0,05$, Tuckey: $p<0,05$. Nas áreas adjacentes à trilha, a cobertura vegetal apresentou valores mais elevados, com média de $47 \%$. Já as parcelas a distâncias de 2 e $10 \mathrm{~m}$ apresentaram-se mais similares, com valores em torno dos $30 \%$ apesar de uma tênue tendência a valores mais altos nas parcelas a $2 \mathrm{~m}$ de distância. Quanto à riqueza, parcelas adjacentes à trilha apresentaram valores maiores, podendo atingir até quatorze espécies diferentes em uma mesma área de $0,25 \mathrm{~m}^{2}$. Já a distâncias de 2 e $10 \mathrm{~m}$, os valores apresentam-se inferiores e bastante semelhantes entre si, em torno de 4 espécies por parcela. O número de indivíduos presentes em cada parcela apresentou valores maiores nas parcelas à beira da trilha, podendo atingir 122 indivíduos. Quanto às distâncias de 2 e $10 \mathrm{~m}$, apesar de alguns valores discrepantes superarem o valor de parcelas à beira da trilha, os valores se comportaram de maneira semelhante, em torno de 14 indivíduos.

Por outro lado, em contraposição aos resultados encontrados por Boucher et al. (1991), as variáveis estudadas não apresentaram diferenças significativas entre um lado e outro da trilha, apesar da tendência mostrada para a cobertura vegetal, que apresentou valores levemente maiores em parcelas situadas ao lado direito da trilha, em média de $36 \%$, comparado aos $32 \%$ do lado esquerdo. A riqueza encontrada em ambos os lados é em média de cinco espécies. O número de indivíduos em parcelas de ambos os lados é semelhante, em torno de 25 indivíduos.

A Figura 3 mostra a proporção de indivíduos de cada grupo, proporção de morfoespécies e a distribuição das duas espécies predominantes, nas três distâncias estudadas.

As plantas amostradas foram agrupadas em 37 famílias e 110 morfoespécies. Plântulas foram separadas de acordo com o tamanho, variando de 1 a $20 \mathrm{~cm}$. A família mais

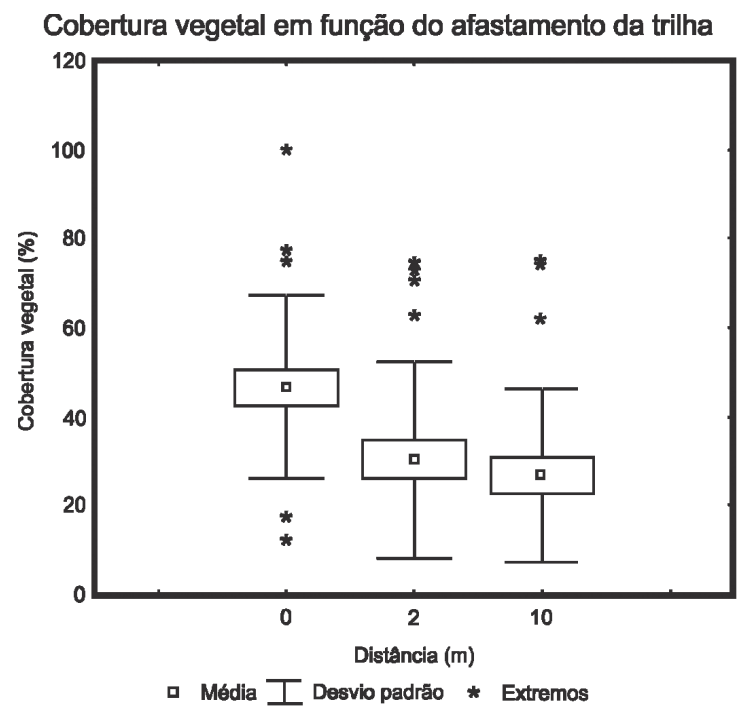

Riqueza em função do afastamento da trilha

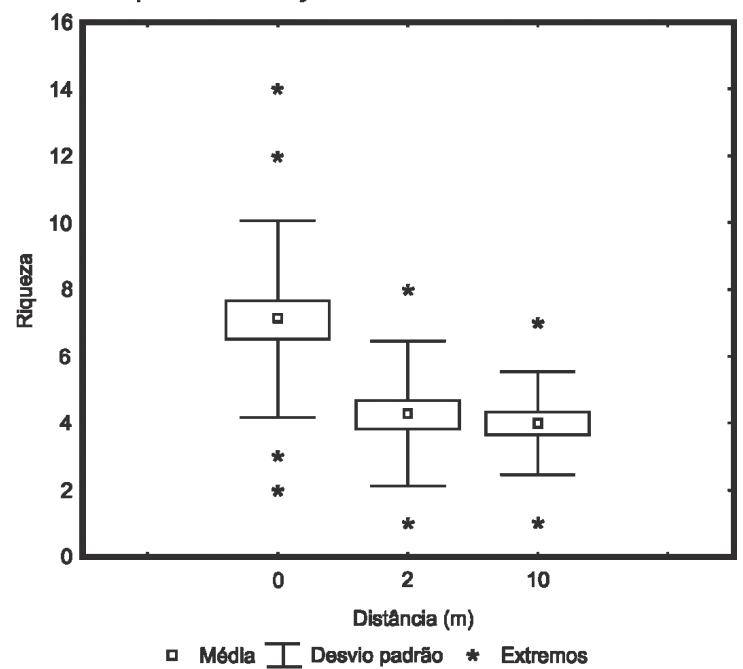

Número de indivíduos em função do afastamento da trilha

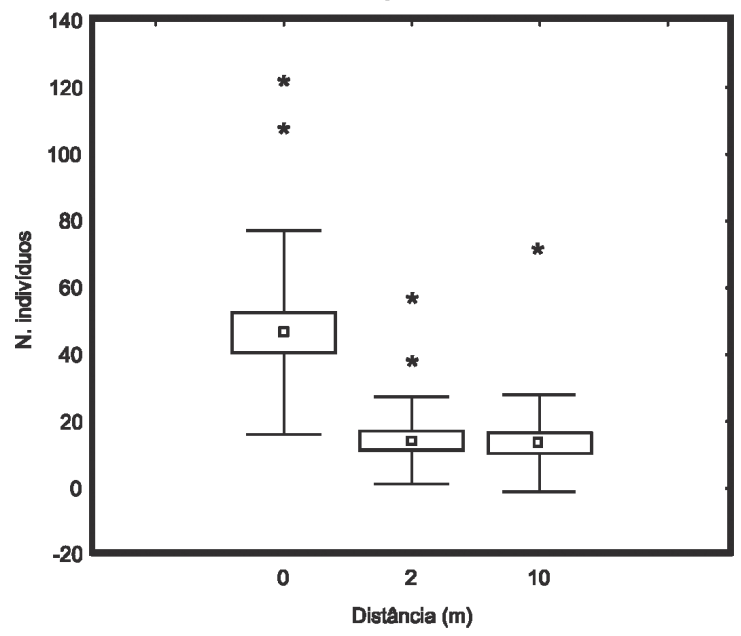

Figura 2. Cobertura vegetal (topo.); Riqueza. (centro); e Número de indivíduos (base), encontrados nas unidades amostrais a 0,2 e 10 metros de distância da trilha. 


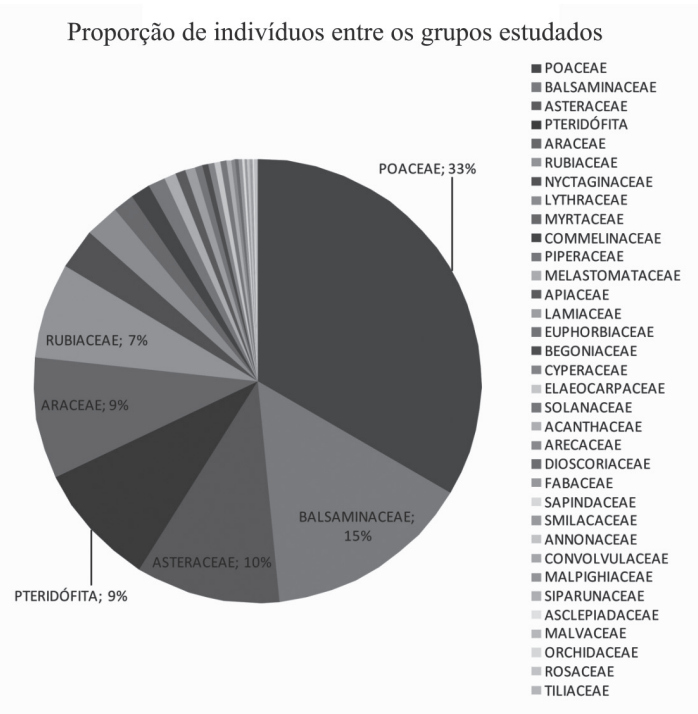

Número de indivíduos das morfoespécies mais abundantes

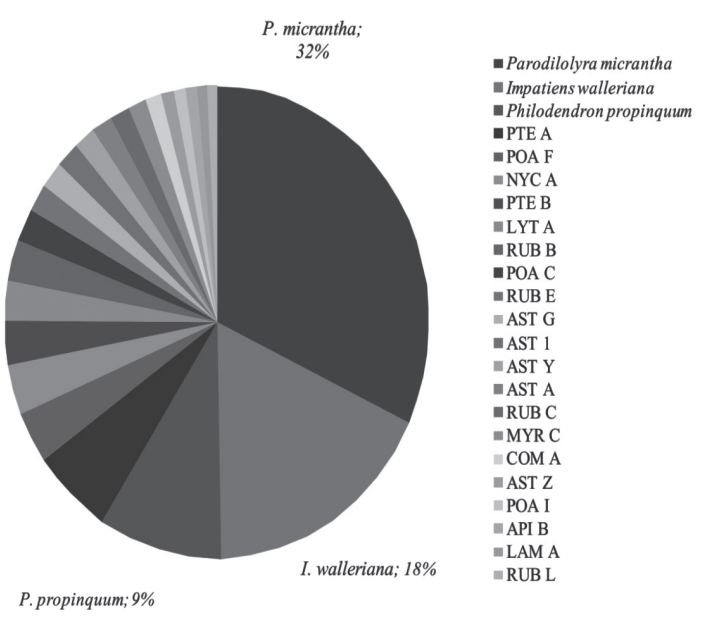

Distribuição das espécies mais frequentes

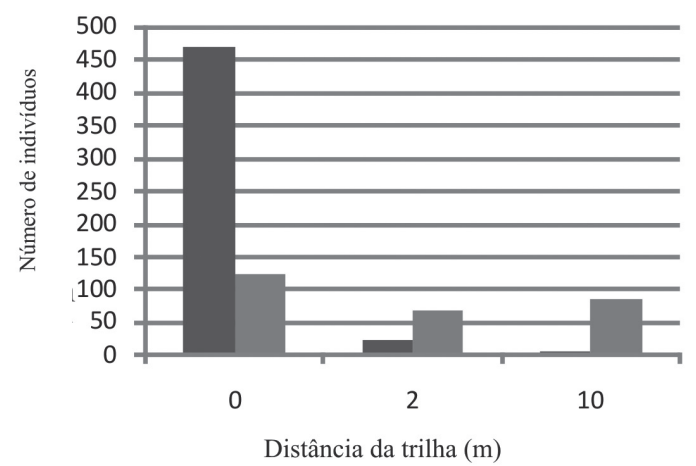

- Parodiolyramicrantha Impatiens walleriana

Figura 3. Incidência de indivíduos de cada grupo/família (topo), proporção entre indivíduos das morfoespécies mais incidentes (centro) e,distribuição das três espécies predominantes nas diferentes distâncias em relação à trilha estudadas (base). representativa foi Poaceae, com $33 \%$ dos indivíduos identificados. Outras famílias de relevante importância no estudo foram Balsaminaceae (15\%) e Asteraceae (10\%). As espécies Parodiolyra micrantha Kunth Davidse \& Zuloaga (Poaceae) e Impatiens walleriana Hook. F. (Balsaminaceae) foram predominantes representando aproximadamente metade do total amostrado. Apesar de ocorrerem em ambientes mais distantes, a $10 \mathrm{~m}$ da trilha, verificou-se que estas espécies predominantes são mais freqüentes em ambientes de borda de trilha $(0 \mathrm{~m})$, especialmente $P$. micrantha.

\section{Discussão}

Foi possível detectar alterações significativas na estrutura da comunidade vegetal, indicando que as áreas mais próximas à Trilha do Mirante apresentam uma dinâmica distinta, com maior cobertura vegetal, maior riqueza e mais indivíduos do que áreas semelhantes localizadas em áreas de menor acesso, menos suscetíveis ao impacto freqüente da visitação.

Esperava-se que as perturbações geradas pelas atividades humanas reduzissem a biomassa e a diversidade da comunidade vegetal a elas sujeita, mas o presente estudo mostrou o contrário (Cole 1978; Hammitt \& Cole 1998; Roncero-Siles 2003). Os valores observados podem decorrer da invasão dos novos hábitats formados à borda da trilha por indivíduos mais competitivos e favorecidos pelas condições ambientais ali oferecidas (Dale \& Weaver 1974; Bright 1986; Roovers et al. 2004).

A vegetação amostrada destacou famílias e espécies com características favoráveis ao ambiente impactado pelas atividades humanas, como alta capacidade de regeneração ou reprodução vegetativa (como $P$. micrantha e $P$. propinquum), maior afinidade à luz (como acontece com a maior parte dos indivíduos de Asteraceae [Junior et al. 2005]), e alta capacidade de dispersão de sementes, como é o caso de Impatiens waleriana. Segundo Campos et al. (2007), esta última é uma espécie herbácea ornamental, invasora, originaria da África e floresce o ano todo. Descrevem-na como heliófita, holoepifita facultativa, com polinização por melitofilia e dispersão por autocoria. A I. walleriana está entre as plantas ornamentais amplamente conhecidas que ocorrem em Floresta Ombrófila Densa, Floresta Ombrófila Mista e Formações Pioneiras de Influência Fluvial. Por conta todas estas características, esta planta encontra-se na lista oficial de espécies vegetais exóticas invasoras do município de São Paulo (Portaria no 19/SVMA 2010, que complementa a Portaria no 154/SVMA/2009).

Estes resultados mostram a necessidade de medidas de manejo da trilha, tais como o controle no número de visitantes, remoção das espécies invasoras, entre as medidas mais urgentes.

Estudos como estes são necessários para dar base a futuras tomadas de decisão no que tange ao planejamento e o manejo das trilhas (Cole \& Landres 1996), visto que as 
medidas de manejo costumam ser baseadas em estudos intuitivos, sem embasamento a respeito dos processos ecológicos (Cole 1978). Futuros estudos com enfoque analítico, como este, podem acrescentar informações a respeito das alterações ambientais em decorrência do uso.

\section{Agradecimentos}

Administração do Parque Natural Municipal Nascentes de Paranapiacaba (PNMNP) por autorizar a realização do trabalho na Unidade, Fundação Santo André por disponibilizar o alojamento; Conselho Nacional de Desenvolvimento Científico e Tecnológico (CNPq) pela bolsa PIBIC; Instituto de Botânica de São Paulo pelo apoio (em especial à Dra. Marie Sugiyama por auxiliar na identificação botânica); Marcel Barranco e Karina Tatit por contribuírem na coleta de dados em campo.

\section{Referências bibliográficas}

Aspinall, R.J. \& Pye, A.M. 1987. The effect of trampling on limestone grassland in the Malham area of North Yorkshire. Journal of Biogeography 14: 105-115.

Boucher, D.H.; Aviles, J.; Chepote, C.; Domínguez Gil, O. E. \& Vilchez, B. 1991. Recovery of trailside vegetation from trampling in a Tropical Rain Forest. Environmental Management 15: 257-262.

Brasil. Ministério do Meio Ambiente. 1998. Primeiro relatório nacional para a Convenção sobre Diversidade Biológica: Brasil. Brasília. 283 p. Disponível em: <http://www.mma.gov.br/port/sbf/chm/relato.html>. (Acesso em 11/11/2008).

Bright, J. 1986. Hiker impact on herbaceous vegetation along trails in an evergreen woodland of Central Texas. Biological Conservation 36: 53-69.

Campos, N.R.; Siqueira, P. \& Voltolini, J.C. 2007. Abundancia da planta invasora Maria-sem-vergonha (Impatiens walleriana) em trilhas com diferentes níveis de uso turístico, Caxambu - MG. Anais do VIII Congresso de Ecologia do Brasil, 23 a 28 de Setembro de 2007, Caxambu - MG

Cole, D.N. 1987. Estimating the susceptibility of wildland vegetation to trailside alteration. Journal of Applied Ecology 15: 281-286.

Cole, D.N. \& Landres, P.B. 1996. Threats to Wilderness Ecosystems: Impacts and Research Needs. Ecological Applications 6: 168-184.

Dale, D. \& Weaver, T. 1974. Trampling effects on vegetation of the trail corridors of North Rocky Mountain Forests. Journal of Applied Ecology 11: 767-772.

Hammitt, W.E. \& Cole, D.N. 1998. Wildland recreation: ecology and management. 2. ed. New York, J. Wiley.

Junior, K.V.; Bordignon, L. \& Diniz, S. 2005. Variação da abundância populacional, riqueza e distribuição de espécies de asteraceae em relação ao tempo decorrido pós-queimada em áreas de cerrado na Chapada dos Guimarães - MT, Brasil. In: Anais do VII Congresso de Ecologia do Brasil. Caxambu, Sociedade Brasileira de Ecologia.

Kinker, S.M.S. 1999. O ecoturismo como uma das estratégias para a conservação da natureza em parques nacionais brasileiros: estudo de caso dos Parques Nacionais de Aparados da Serra e Caparaó. Dissertação (Mestrado) - PROCAM. São Paulo, Universidade de São Paulo, Ciência Ambiental.

LUME. 1999. Laboratório de Urbanismo da Metrópole da Faculdade de Arquitetura e Urbanismo da USP. Plano de desenvolvimento sustentável da Vila de Paranapiacaba. Ed. USP, São Paulo.

Roncero-Siles, M.F. 2003. Modelagem espacial para atividades de visitação pública em áreas naturais. 2003. 147f. Dissertação (Mestrado em Ecologia) - Instituto de Biociências, Universidade de São Paulo, São Paulo. (http://www.teses.usp.br/teses/disponiveis/41/41134/tde12042009-214021/pt-br.php)

Roovers, P.; Baeten, S. \& Hermy, M. 2004. Plant species across path ecotones in a variety of common vegetation types. Plant ecology 170: 107-119.

Santo André. 2008. Prefeitura de Santo André. Atlas do Parque Natural Municipal das Nascentes de Paranapiacaba: Desvendando um Novo Parque. Santo André: Prefeitura de Santo André.

Sokal, R.R. \& Rohlf, F.J. 1969. Biometry. The principles and practices of statistics in biological research. San Francisco, Freeman and Co.

Sun, D. \& Walsh, D. 1998. Review of studies on environmental impacts of recreation and tourism in Australia. Journal of Environmental Management 53: 323-338. 